\title{
Möglichkeiten und Grenzen der Gestaltung von Eheverträgen*
}

Manche Entscheidungen des Bundesverfassungsgerichts erregen mehr als andere die Gemüter und rufen Applaus wie Kritik hervor. Zu ihnen zählen zwei aus dem Jahre 2001 zur Inhaltskontrolle von ehevertraglichen Vereinbarungen. Glaubt man den Worten Münchs, ${ }^{1}$ dann haben sie gleich einem Paukenschlag auf die bis dahin gefestigte familiengerichtliche Rechtsprechung zu dieser Frage gewirkt. Die Resonanz darauf war jedenfalls nachhaltig, aber auch vielfältig.

Einerseits wird begrüßt ${ }^{2}$ die Entscheidungen hätten mit dankenswerter Deutlichkeit klargestellt, dass auch Eheverträgen ihre Grenzen gesetzt werden müssen, ${ }^{3}$ um eindeutig untragbare Ergebnisse auszuschließen. ${ }^{4}$ Damit sei die Rechtsprechung aus der kühlen Epoche »voller Vertragsfreiheit « in die allerdings sehr viel unübersichtlichere Gegenwart materialer Vertragsgerechtigkeit befördert worden. ${ }^{5}$ Andererseits werden die Entscheidungen als Ärgernis empfunden. Sie erinnerten an längst überwundene Vorstellungen vom Wesen der Ehe. ${ }^{6}$ Mit ihnen werde in gefährlicher Weise der soziale Schutzgedanke gegen die Freiheitsidee gestellt, ${ }^{7}$ was nicht zu einer eigenverantwortlichen Lebensgestaltung in einer freien Gesellschaft passe. Außerdem erweise das Gericht den Frauen einen Bärendienst. ${ }^{8}$ Ja, selbst Faust und Gretchen werden bemüht, um Zuspruch wie Kritik zu untermalen. ${ }^{9}$

Der Frage, auf welchem geistigen Humus die Entscheidungen fußen, hat sich in jüngster Zeit auch Bergschneider gewidmet. Seine Analyse führt zu dem Ergebnis, das Bundesverfassungsgericht habe bei diesen Entscheidungen rechtsphilosophische Tendenzen aus jüngerer Zeit und dabei insbesondere Teile der Philosophie von Habermas, aber auch von Rawls in seine Judikatur aufgenommen. ${ }^{10}$ Die von Habermas begründete Diskurstheorie setze bei der argumentativen Suche nach plausiblen und somit für alle Betroffenen tragbaren Ergebnissen vornehmlich auf die Institutionalisierung von Kommunikationsvoraussetzungen und Diskursverfahren, die in der Anerkennung der Gleichwertigkeit und dem Ernstnehmen aller Gesprächsteilnehmer lägen. Wenn das Bundesverfassungsgericht in seinen Entscheidungen von der Vermeidung einer auf ungleichen Verhandlungspositionen beruhenden Dominanz eines Ehepartners, der

* Vortrag auf der 1. Jahresarbeitstagung des Notariats am 18. September 2003 in Würzburg (ohne Berücksichtigung nachträglicher Rechtsänderungen).

1 MittBayNot 2003, S. 107.

2 Schwab, FamRZ 2001, S. 349.

3 Büttner, FF 2001, S. 65.

4 Schwab, a.a.O.

5 Dauner-Lieb, AcP 201 (2001), S. 295 (305).

6 Rauscher, DNotZ 2002, S. 751 (752).

7 Rauscher, FuR 2001, S. 155.

8 Röthel, NJW 2001, S. 1334 (1335).

9 Büttner, FamRZ 1997, S. 600; Grziwotz, FamRB 2002, S. 58 (60).

10 Bergschneider FF 203, S. 118 (119). 
Situation von Unterlegenheit oder der Schwächung einer Verhandlungsposition rede, dann handele es sich um diesen theoretischen Ansatz im juristischen Begriffskleid, bei dem die vom Gericht verlangte richterliche Inhaltskontrolle der dem Staat von Habermas zugewiesenen Aufgabe der Institutionalisierung diskursiver Verfahren entspreche. Hierin drücke sich eine verbreitete, tiefgehende rechtsphilosophische Haltung aus, die auf allgemeinen Demokratisierungsvorstellungen beruhe, als deren weiterer Repräsentant vor allem auch Rawls zu nennen sei.

Das Bundesverfassungsgericht also als Transporteur demokratietheoretischer Vorstellungen in das Feld familiärer Individualbeziehungen? Nun tut es bestimmt nicht nur Verfassungsrichtern gut, sich auch mit der Rechtsphilosophie beziehungsweise Rechtstheorie auseinander zu setzen. Und Habermas ist hier durchaus ein Protagonist. Allerdings gibt es in seinem von Bergschneider zitierten Werk »Faktizität und Geltung « andere Passagen, die meines Erachtens einschlägiger auf das Problem hinweisen, mit dem es das Bundesverfassungsgericht bei seinen Entscheidungen zu tun hatte. Ging es doch nicht um die Frage, wie Legitimation von Recht in unserem Verfassungsstaat entsteht, sondern grundsätzlich darum, was Privatautonomie im Verfassungsstaat bedeutet. Unter dem Stichwort der »Materialisierung des Privatrechts « beschreibt hierzu Habermas ${ }^{11}$ nicht nur den von vielen immer noch als schmerzhaft empfundenen historischen Auflösungsprozess der angenommenen Autarkie des Privatrechts hin zu dessen Einbindung in das Verfassungsrecht, womit auch im Privatrecht, wie es Konrad Hesse einmal formuliert hat, ${ }^{12}$ die Gewährleistung der Existenz der Rechtsgenossen und der Schutz des Schwächeren gleichen Rang wie die Verfolgung der eigenen Interessen erfahren hat. Habermas weist zudem darauf hin, dass sich die Einschränkung der klassischen Grundfreiheiten unter den geänderten Vorzeichen keineswegs allein auf die Interferenz anderer Rechtsprinzipien zurückführen ließe. Was als Einschränkung erscheine, sei nur die Kehrseite der Durchsetzung gleicher subjektiver Handlungsfreiheiten für alle, denn Privatautonomie im Sinne dieses allgemeinen Freiheitsrechts impliziere ein allgemeines Gleichheitsrecht. Wenn sich daraus für eine der Parteien tatsächliche Einschränkungen gegenüber dem Status quo ante ergäben, handele es sich nicht um normative Einschränkungen des Prinzips rechtlicher Freiheit, sondern um die Abschaffung solcher Privilegien, die mit der von diesem Prinzip geforderten Gleichverteilung subjektiver Freiheiten unvereinbar seien. ${ }^{13}$

Damit sind wir schon mitten im Thema, das in seiner Grundsätzlichkeit wahrlich nicht erst seit den als anstoßend wie anstößig empfundenen Entscheidungen des Bundesverfassungsgerichts und nicht erst durch Habermas philosophisch und soziologisch, aber auch staatsrechtlich und zivilrechtlich dogmatisch behandelt worden ist.

Schon Max Weber führte in seiner Rechtssoziologie die Begrenzungen für die Vertragsfreiheit und ihre Gründe auf. Für das Zurückdrängen der »sexuellen Vertragsfreiheit«, wie er es nannte, machte er im Übrigen nicht das damalige Verfassungsrecht, sondern die Furcht vor einer Gefährdung der Erziehungschancen der Kinder, die auto-

11 Faktizität und Geltung, S. 478.

12 Hesse, Verfassungsrecht und Privatrecht 1988, S. 34.

13 Habermas, a.a.O., S. 484. 
ritären Instinkte von Männern, die Geschlechtseitelkeit der Frauen und die Sorge um eine entsprechende Position innerhalb der Familie verantwortlich. ${ }^{14}$ Wieacker war es, der prägnant herausgearbeitet hat, dass das Bürgerliche Gesetzbuch zwar als spätgeborenes Kind des klassischen Liberalismus entstanden ist, das aber sein Sozialbild, auf dem es fußte, schon bei seiner Entstehung nicht mehr trug. Er pries es damals als Errungenschaft der Rechtsprechung, die formale Freiheitsethik, die der deutschen Privatrechtsordnung zugrundeliege, in eine materiale Ethik sozialer Verantwortung zurückverwandelt $\mathrm{zu}$ haben, so zum Beispiel auch mit Hilfe einer immer stärkeren Ausnutzung der Sittenwidrigkeitsklausel. ${ }^{15}$ Und er mahnte an, dass eine selbstbewusste und öffentlich verantwortliche Privatrechtswissenschaft nur notwendig und möglich bleibe, wenn sie ihre gesellschaftliche Wirklichkeit wahrnehme und forme. Auch Raiser hob in einer Rede vor dem Juristentag die veränderte Rolle des Privatrechts im demokratischen Verfassungsstaat hervor: nicht eine vom Staat distanzierte oder von ihm aufgesogene, sondern eine bei Wahrung ihrer Selbständigkeit in den Staat integrierte Privatrechtsgesellschaft und damit eine der Verfassung verpflichtete Privatrechtsordnung gelte es zu verwirklichen. ${ }^{16}$ Auf die Vertragsfreiheit transponiert wies nicht erst Rawls, sondern schon Esser darauf hin, dass diese die Eröffnung der Chance bedeute, seine Interessen durchzusetzen, aber auch die Gefahr, dass der Mächtigere die Vertragsbedingungen nach seinem Ermessen festsetze. Für das Recht erwachse daraus die doppelte Aufgabe, ungeachtet vorhandener Chancenungleichheit ein zureichendes Maß an gerechter Güterverteilung zu gewährleisten und Missbräuche der Vertragsfreiheit, auch durch richterliche Vertragskontrolle, zu verhindern. ${ }^{17}$ Schauen wir schließlich auf jüngste zivilrechtsdogmatische Versuche einer Ortung der Privatautonomie in den komplexen gesellschaftlichen Kontexten einer globalisierten Welt, dann wiederholt sich auch hier die Frage nach der Notwendigkeit einer Implementierung von verfassungsrechtlichen Maßstäben in selbstreferenzielle zivile Teilbereiche. So spricht zum Beispiel Teubner von einem Prozess juridischer Entfesselung, die einher zu gehen habe mit einer Zügelung systemspezifischer Rationalität. Es gehe insofern um die institutionelle Dimension der Grundrechte in privaten Gesellschaftsbereichen. Dabei würden gesellschaftliche Normierungen im Zentrum des Rechts akzeptiert. Allerdings seien in einem Prozess gerichtlicher Kontrolle dieses privaten Rechts korrumpierende Elemente abzuwehren, die aus den Rechtseffizienzen der externen Rechtsquellen stammten. ${ }^{18}$ Dabei versteht er unter Korruption die strukturelle Unverträglichkeit der Rationalität des privat gesetzten Rechts mit der Rationalität gesellschaftlicher Normbildungen, die von der Verfassung geprägt sind.

Dieser kurze Ausflug in die Rechtstheorie sollte zunächst einmal illustrieren, dass das Bundesverfassungsgericht mit seinen Ausführungen zu den Grenzen der Vertrags-

14 Max Weber, Rechtssoziologie, S. 158.

15 Wieacker, Das Sozialmodell der klassischen Privatrechtsgesetzbücher und die Entwicklung der modernen Gesellschaft, S. 24 f.

16 Raiser, Grundgesetz und Privatrechtsordnung 1967, S. B 31, 46. DJT.

17 Esser, Schuldrecht Allgemeiner Teil, 3. Aufl., S. 81.

18 Teubner, Globale Zivilverfassungen: Alternativen zur staatszentrierten Verfassungstheorie in: ZaöRV 2003, Band 63, S. 22. 
freiheit nicht allein steht, sondern sich in einem breiten Diskussionszusammenhang der Verfassungs- und Zivilrechtslehre wiederfindet. Deshalb drängt sich nicht so sehr die Frage auf, ob das Gericht mit seinen Entscheidungen eine Rolle rückwärts in vergangene Zeiten überholten Schutzdenkens gemacht hat, wie manche meinen, sondern vielmehr die, welchem Bild von »freier « Gesellschaft manche seiner Kritiker nachtrauern, hat doch die ungezügelte Freiheit zu keiner Zeit ein theoretisches, geschweige denn ein gesellschaftliches Fundament besessen. Denn selbst der Vertragsfreiheit, auch in ihrem klassisch-liberalen Verständnis, lag idealtypisch die Vorstellung von zwei Gleichen als Vertragsschließenden zugrunde, weil nur so die Annahme eines gerechten Interessenausgleichs gestützt werden konnte, mit der sie ihre Rechtfertigung fand.

Nun geht es aber bei den Entscheidungen des Bundesverfassungsgerichts zur Vertragsfreiheit bei Eheverträgen nicht um Grenzziehungen im Rahmen allgemeiner Handlungsfreiräume für das gesellschaftliche Miteinander, sondern um solche im familiär ehelichen Gestaltungsraum, also in höchstpersönlichen Beziehungen: um die Freiheit, die Bedingungen eines ehelichen Zusammenlebens zu gestalten und die Freiheit jedes der beiden in Ehe Verbundenen, auf diese Gestaltung Einfluss zu nehmen. Haben hier andere Gesichtspunkte Platz zu greifen und wenn ja, verstärken sie die Notwendigkeit, der Freiheit Grenzen zu setzen oder führen sie dazu, die Intimsphäre freizuhalten von Begrenzungen eigenverantwortlicher Gestaltung? Um hierauf eine Antwort finden zu können, wollen wir uns nun diesem Freiheitsraum nähern, wie ihn unsere Verfassung vorgibt und zugleich schützt, aber auch, wie er vom Einzelnen vor welchem sozialen und gesellschaftlichen Hintergrund genutzt werden kann.

Wie bei der Vertragsfreiheit im Allgemeinen spielt auch bei der Ehevertragsfreiheit zunächst das jeweilige rechtliche und tatsächliche Potenzial zur Durchsetzung eigener Freiheitsräume gegenüber dem anderen Kontrahenten eine Rolle. Eine zusätzliche Prägung erfährt aber das personale eheliche Vertragsverhältnis zwischen Mann und Frau durch das Maß an Gleichberechtigung zwischen den Geschlechtern, das von der Verfassung vorgegeben ist, beziehungsweise in Recht, Gesellschaft und zwischen den Vertragspartnern herrscht. Damit zeichnen sich die verfassungsrechtlichen Bezugspunkte $\mathrm{ab}$, in deren Lichte die Ehevertragsfreiheit zu betrachten ist: Art. 2 Abs. 1 GG, der als Ausfluss der allgemeinen Handlungsfreiheit auch die Vertragsfreiheit schützt, ${ }^{19}$ Art. 6 Abs. 1 GG, der die Ehe unter seinen Schutz stellt ${ }^{20}$ und schließlich Art. 3 Abs. 2 GG, der einfordert, dass die Ehe als gleichberechtigte Partnerschaft zwischen Mann und Frau gelebt werden kann. ${ }^{21}$ Diese Verfassungsnormen bilden zugleich den spezifischen Spannungsbogen zwischen Freiheit und Schutz, in dem ehevertragliche Vereinbarungen stehen: Beidem ist aufgrund der verfassungsrechtlichen Gewährleistung Rechnung zu tragen.

Schauen wir uns unter diesen Vorzeichen einmal an, wie der Gesetzgeber seinem Auftrag nachgekommen ist, den Ehegatten einerseits Freiheitsräume zu sichern, dabei

19 Vgl. BVerfGE 8, 274 (328).

20 Vgl. BVerfGE 31, 58 (82 f.); 105, 313 (345).

21 Vgl. BVerfGE 35, 382 (408); 105, 1 (10). 
jedem von ihnen die Möglichkeit einzuräumen, die Freiheit auch nutzen zu können und andererseits dafür zu sorgen, dass sie sich als gleichberechtigte Partner gegenübertreten können. Gerade Letzteres tat nach In-Kraft-Treten des Grundgesetzes zunächst bitter Not, war doch das Familienrecht bis dahin nicht nur von der rechtlichen Dominanz des pater familiae geprägt, sondern auch von klaren Rollenzuweisungen, nach denen die Frau an den heimischen Herd gehörte und der Mann der Ernährer der Familie war und damit das Sagen hatte. Hier waren - wie schon im Parlamentarischen Rat deutlich zur Sprache kam - gewaltige Umbauarbeiten angesagt, um der verfassungsrechtlichen Vorgabe nach Gleichberechtigung auch im Familienrecht Genüge zu leisten. Auch wenn sich der Gesetzgeber zunächst nur widerstrebend an dieses Werk setzte und des Öfteren das Ziel verfehlte, so dass er vom Bundesverfassungsgericht zur Korrektur angemahnt werden musste, ${ }^{22}$ kann nunmehr nach jahrzehntelanger Reformarbeit festgestellt werden, dass das Familienrecht einer gleichberechtigten Partnerschaft in der Ehe keine größeren Steine mehr in den Weg legt. Insbesondere ist die Aufgabenverteilung in der Ehe weder rechtlich vorgegeben, noch hat darüber maßgeblich nur einer der Ehegatten zu bestimmen, sondern beide gemeinsam. Das auf Gleichheit beruhende Recht hat also den Ehegatten auch mehr Freiheit gebracht, die sie in Verfolgung ihrer gemeinsamen Interessen nutzen können.

Allerdings ist die Ehe nicht nur der Schlüssel für eine freie Gestaltung der Beziehung zwischen zwei Menschen. Dafür reichte das allgemeine Vertragsrecht aus. Die Ehe ist vielmehr ein Institut, das den rechtlichen Rahmen für eine dauerhafte Lebensgemeinschaft zwischen Mann und Frau bietet,$^{23}$ die ihre Grundlage neben Liebe und Zuneigung in gemeinsamer Verantwortung, wechselseitiger Unterstützung und solidarischem Handeln findet. Und auch in ihr kann sich gleiche Freiheit an den gesellschaftlichen Rahmenbedingungen und tradierten Rollenverständnissen reiben, auf die sie stößt. So kann der Wunsch, sich Erwerbs- und Hausarbeit zu teilen, an mangelnder außerhäuslicher Kinderbetreuung, fehlenden Arbeitsmöglichkeiten, divergierenden Einkunftschancen oder entgegenstehenden Erwartungshaltungen im sozialen und beruflichen Umfeld scheitern. Andererseits schlägt sich die Entscheidung zu einer Ehe, in der die Frau den Haushalt führt und die Kinder betreut, während der Mann erwerbstätig ist, angesichts der gesellschaftlichen, ökonomischen und sonstigen rechtlichen Rahmenbedingungen nicht nur auf die Einkommenshöhe nieder, die das eheliche Leben prägt, sondern verweist den Haushalt führenden Ehegatten zur eigenen Absicherung zunächst ausschließlich auf den familiären Unterhaltsverbund, wird doch über die häusliche Arbeit kein Einkommen erzielt und können deshalb auch grundsätzlich keine eigenen Einkommensersatzansprüche erworben werden.

Um gerade für eine solche Arbeitsaufteilung zwischen den Ehegatten, die jedenfalls in Deutschland zumindest dann, wenn sich Kinder einstellen, immer noch vorherrschend ist ${ }^{24}$ sicherzustellen, dass sie nicht innerhalb des Eheverbundes zu Schieflagen zu Lasten der Hausfrau und in noch geringer Zahl zu Lasten des Hausmanns führt, ist

22 Beginnend von BVerfGE 3, 225.

23 Vgl. BVerfGE 10, 59 (66); 62, 323 (330); 105, 313 (345).

24 Siehe BMFSFJ, Die Familie im Spiegel der amtlichen Statistik, 2003, S. 107 ff. 
vom Gesetzgeber nicht nur klargestellt worden, dass die Leistungen der Haushaltsführung und Kinderbetreuung eines Ehegatten gleichrangig mit der Bereitstellung des familiären Barunterhalts durch den anderen Ehegatten zu bewerten sind. ${ }^{25}$ Der Gesetzgeber hat darüber hinaus mit dem Güterstand der Zugewinngemeinschaft und dem Versorgungsausgleich den Eheleuten Regelungsmodelle zur Verfügung gestellt, die für den Fall der Scheidung die Gleichrangigkeit der während der Ehe erbrachten Leistungen berücksichtigen und deshalb beide Ehegatten gleichermaßen an den während der Ehe erworbenen Vermögenszuwächsen und sozialen Anwartschaften partizipieren lassen. Zugleich hat er dem Ehegatten, der nach der Trennung die Kinder betreut, oder der aufgrund langjähriger Familienarbeit, seines inzwischen eingetretenen Alters und seiner fehlenden Möglichkeiten, wieder im Erwerbsleben Fuß zu fassen, einen Unterhaltsanspruch gegenüber dem früheren erwerbstätigen Ehegatten eingeräumt. Allerdings hat es der Gesetzgeber den Ehegatten generell freigestellt, über ihre ehelichen und nachehelichen Verhältnisse hiervon abweichende Regelungen zu treffen, um damit im Sinn von Art. 6 Abs. 1 und Art. 2 Abs. 1 GG den Ehegatten zu ermöglichen, ihr eheliches Leben und dessen nacheheliche Wirkungen ihren Vorstellungen von Arbeitsteilung in der Ehe im beiderseitigen Interesse zu regeln. Hier nun beginnt das Feld der ehevertraglichen Freiheit mit dem daraus fließenden Recht, seinen Ehe- und damit Vertragspartner selbst zu wählen und den Inhalt des Vertrages selbst zu bestimmen. ${ }^{26}$

Nun wird aber auch die Vertragsfreiheit durch die Verfassung nicht grenzenlos gewährt, sondern findet ihre Schranken in den Rechten anderer sowie der verfassungsmäßigen Ordnung. ${ }^{27}$ Zudem ist zu berücksichtigen, dass dieses Freiheitsrecht beide Vertragspartner für sich reklamieren können, die aber durchaus unterschiedlicher Stellung sein, verschiedene Interessen haben und gegenläufige Ziele verfolgen können. Soll sich im Vertrag ein freies und eigenverantwortliches Handeln beider Vertragspartner und darüber ein angemessener Interessenausgleich zwischen ihnen verwirklichen, dann bedarf es nicht nur des Raums zur freien Gestaltung. Vielmehr ist Voraussetzung, dass beide Vertragspartner auch in der Lage sind, selbstbestimmt zu handeln, oder wie es das Verfassungsgericht ausgedrückt hat, dass die Bedingungen der Selbstbestimmung des Einzelnen auch tatsächlich gegeben sind. ${ }^{28}$ Ist dies nicht der Fall, perveriert Vertragsfreiheit zum Recht auf Bestimmung über einen anderen und verletzt dessen Recht, seine Interessen in einen Ausgleich mit denen seines Vertragspartners zu bringen. Die kollidierenden Grundrechtspositionen sind also in ihrer Wechselwirkung zu sehen und so zu begrenzen, dass sie für alle Beteiligten möglichst weitgehend wirksam werden. ${ }^{29}$ Einem solchen Ausgleich konkurrierender Grundrechtspositionen dient letztlich auch die richterliche Vertragskontrolle über die zivilrechtlichen Generalklauseln, die - wie das Bundesverfassungsgericht schon in seiner berühmten Lüth-Entscheidung formuliert hat - als »Einbruchsstellen« der Grund-

25 So $\S ~ 1360,1606$ Abs. 3 Satz 2 BGB; vgl. auch BVerfGE 105, 1 (11).

26 Vgl. BVerfGE 80, 81 (92).

27 So Art. 2 Abs. 1 GG.

28 Vgl. BVerfGE 81, 242 (254 f.); 103, 89 (100).

29 Vgl. BVerfGE 103, 89 (100). 
rechte im Privatrecht dienen und das Medium sind, über das die Grundrechte dort ihre Wirkung entfalten. ${ }^{30}$ Dabei hat zunächst grundsätzlich zu gelten, dass der im Vertrag zum Ausdruck kommende übereinstimmende Wille der Vertragspartner einen für sie sachgerechten Interessenausgleich hergestellt hat. Ist jedoch aufgrund einer besonders einseitigen Aufbürdung von vertraglichen Lasten und einer erheblich ungleichen Verhandlungsposition der Vertragspartner ersichtlich, dass in einem Vertragsverhältnis ein Partner ein solches Gewicht hat, dass er den Inhalt des Vertrages faktisch einseitig bestimmen kann, dann ist dem auch dort, wo der Gesetzgeber von spezifischen Schutzregelungen im Vertragsrecht abgesehen hat, über die zivilrechtlichen Generalklauseln, die insofern als Untermaßverbote wirken, Einhalt zu gebieten. Dies gilt, wie das Bundesverfassungsgericht nunmehr im 103. Bande klargestellt hat, nicht nur für Verträge im allgemeinen Rechtsverkehr, bei denen sich mit unterschiedlicher ökonomischer und gesellschaftlicher Macht ausgestattete Vertragspartner gegenüberstehen, sondern auch für Eheverträge. Auch der Freiheit der Ehegatten, mit Hilfe von Verträgen die ehelichen Beziehungen und wechselseitigen Rechte und Pflichten zu gestalten - so heißt es in der Entscheidung -, hat der Staat dort Grenzen zu setzen, wo der Vertrag nicht Ausdruck und Ergebnis gleichberechtigter Lebenspartnerschaft ist, sondern eine auf ungleichen Verhandlungspositionen basierende einseitige Dominanz eines Ehegatten wiederspiegelt. ${ }^{31}$ Hier ist es Aufgabe der Gerichte, über die zivilrechtlichen Generalklauseln den Inhalt des Vertrages einer Kontrolle zu unterziehen und gegebenenfalls zu korrigieren. Anlass für die Inhaltskontrolle eines Ehevertrages ist demnach seine am Maßstab der Gleichberechtigung gemessene, unausgewogene Rechte- und Lastenverteilung im Verhältnis zur Lebenslage der Ehegatten und zu den Aufgaben, die ihnen in der Ehe beiderseitig zugedacht sind.

Für die zu entscheidenden Fälle des Vertragsschlusses mit einer Schwangeren hat das Gericht dann dargestellt, worin im Allgemeinen die Unterlegenheit einer Schwangeren gegenüber dem Erzeuger ihres Kindes liegt, wenn mit einem Vertrag die Bedingungen für einen Eheschluss verhandelt werden. Es hat deutlich gemacht, dass die Schwangerschaft nur ein Indiz für eine vertragliche Disparität ist und als weitere maßgebliche Faktoren für die Einschätzung des Kräfteverhältnisses zwischen den (künftigen) Ehegatten die jeweilige Vermögenslage, die berufliche Qualifikation und Perspektiven sowie die von den Ehegatten ins Auge gefasste oder praktizierte Aufteilung von Erwerbs- und Familienarbeit genannt. ${ }^{32}$ Des Weiteren hat es ausgeführt, dass auch die Frage, ob der Inhalt des Ehevertrages einen der beiden Ehegatten einseitig belastet, nicht losgelöst von der familiären Konstellation beantwortet werden kann, die die Vertragspartner anstreben oder in der sie sich befinden. Ein Verzicht auf einen nachehelichen Unterhalt trifft zum Beispiel einen auch während der Ehe berufstätigen und über ausreichendes Einkommen oder Vermögen verfügenden Ehegatten ersichtlich weniger als den, der sich nach dem Willen beider Ehegatten in der Ehe ausschließlich der Haushaltsführung und Kinderbetreuung widmet, ist für ihn damit doch einerseits

30 BVerfGE 7, 198 (205 f.).

31 Vgl. BVerfGE 103, 89 (101).

32 Vgl. BVerfGE a.a.O., S. 104. 
der Verlust von eigenem Einkommen während der Ehe verbunden, was ihn auf Unterhaltsleistungen durch den Ehepartner angewiesen macht. Und andererseits verschlechtern sich seine Chancen nach der Ehe, sich selbst wieder angemessen unterhalten zu können.

Wenn sich aber ungleichgewichtige Lagen der Ehepartner nicht nur im Vertragsinhalt wiederspiegeln, sondern dieser zugleich ihr ungleiches Kräfteverhältnis zementiert, wenn ein Ehevertrag gerade dort einem Ehepartner die Unterstützung versagt, wo er sie aufgrund der gemeinsam beschlossenen ehelichen Aufgabenverteilung in besonderem Maße braucht, kann sein Inhalt nicht mehr die Annahme eines angemessenen Interessenausgleichs tragen, denn er widerspricht nicht nur dem gesetzlichen Schutz, der in Erfüllung des Auftrags aus Art. 3 Abs. 2 GG gerade derartige Schieflagen zwischen den Ehegatten verhindern soll, sondern setzt das außer Kraft, was die Ehe auszeichnet: Verantwortung auch für den anderen zu tragen, wenn dieser darauf angewiesen ist.

Dies hat nichts mit dem Sittenbild des 19. Jahrhunderts zu tun, wie manche Kritiker dem Bundesverfassungsgericht vorgehalten haben, sondern mit dem Sinn, der der Ehe bei aller Vielfalt ihrer Ausgestaltung und allem Wandel, den sie erfahren hat, auch heute noch innewohnt. ${ }^{33}$ Und das Gericht ist damit auch keinem Klischee von der schwachen Frau, die zum Schicksal des Kinderkriegens geschlagen ist, und dem starken schwängernden Mann, der sich vor der Verantwortung drückt und zur Heirat getragen werden müsse, aufgesessen, mit dem, wie kritisiert wird, die Frau in die Nähe struktureller Geschäftsunfähigkeit gestellt werde. So amüsant diese Karikatur, so schwach ist sie in der Analyse ihres Gegenstandes und damit diesem so fern. Es kann wohl nicht geleugnet werden, dass gerade das Bundesverfassungsgericht in der Tradition seiner Entscheidungen steht, die Gleichberechtigung der Geschlechter zu unterstützen und dabei von Schwarz-Weiß-Malereien abzusehen. Dies ist auch Richtschnur der Ehevertragsentscheidungen gewesen, die gerade dazu auffordern, von abstrakten Geschlechtsrollenzuweisungen abzulassen und genauer auf die konkreten Lebensumstände derjenigen zu blicken, die sich ehevertraglich binden, um danach zu differenzieren. Tut man dies aber, wird man nicht übersehen können, dass es neben persönlichen Unterschieden, zum Beispiel die Herkunft, die Qualifikation, das Einkommen oder das Alter betreffend, auch unterschiedliche Situationen und Konstellationen gibt, die für die Bewertung eine Rolle spielen, ob jemand im Kräftespiel des vertraglichen Aushandelns als alleiniger Verlierer herauskommt. Und niemand erklärt eine Ehefrau, die im Einvernehmen mit ihrem Ehemann keiner Erwerbsarbeit nachgeht, weil sie den Haushalt bestellt und die Kinder betreut, für unmündig, wenn auf ihre finanzielle Abhängigkeit vom Mann hingewiesen wird, die sich auch dann fortsetzen kann, wenn die Ehe scheitert und ihr der Weg zur eigenen Absicherung wegen der Kinder oder des Alters verbaut ist. Wenn Rauscher hier meint, das Begehren, wirtschaftliche Solidarität in der Ehe nur so lange zu leisten, wie emotionale Solidarität besteht, sei in keiner Weise ethisch zu beanstanden, dann verkennt er, dass dies zwar gelten mag für diejenigen, die sich vor und während der Ehe die Voraussetzungen für eine ökonomische

33 Vgl. BVerfGE 31, 58 (82 f.); 105, 313 (345). 
Entsolidarisierung nach der Ehe erhalten oder geschaffen haben, nicht aber für die, die in der Ehe zwar ihr Glück finden und Arbeit erbringen, aber finanzielle Unabhängigkeit und Chancen auf eigene Absicherung verlieren. Außerdem geht es nicht um Ethik oder Moral, sondern vorrangig darum, wo unsere Verfassung der Vertragsfreiheit des einen Ehepartners Grenzen setzt, um zu verhindern, dass der andere Ehegatte seiner auch ihm von der Verfassung geschützten Rechte verlustig geht.

Allerdings gibt es durchaus ernst zu nehmende Zweifel, ob es richtig ist, die Notwendigkeit einer Inhaltskontrolle und gegebenenfalls Korrektur auch von Eheverträgen vorrangig an der mangelnden Vertragsparität und Selbstbestimmung der Vertragspartner festzumachen, und zwar deshalb, weil gerade in einer Intimbeziehung die Festlegung der konkreten Opfergrenze schwer an Verallgemeinerungen zu messen ist. Daraus aber zu folgern, solche Verträge seien einer Inhaltskontrolle gänzlich entzogen, ließe außer Acht, dass es gerade die Besonderheit dieser personalen Beziehung ist, die der Vertragsfreiheit dort Grenzen setzt, wo sich ihre eigene Handlungslogik nicht mehr mit der eines kalkulierenden ökonomischen Tauschgeschäfts verträgt. ${ }^{34}$ Das rationale Kalkül, das beim Vertrag unterstellt wird, bricht sich dort, wo Nähe herrscht und der Blick sich nicht allein auf die eigenen Interessen richtet, sondern auch denen der anderen in der persönlichen Beziehung gilt. Damit stellt sich die Frage der Geltungsbereiche wirtschaftlicher Rationalität und familialer Solidarität, wie es Teubner formuliert hat. ${ }^{35} \mathrm{Da}$ aber nicht nur die Vertragsfreiheit, sondern auch die eheliche Beziehung unter den Schutz der Verfassung gestellt ist, ist dafür Sorge zu tragen, dass durch Nutzung der Vertragsfreiheit im sozialen ehelichen Verbund nicht die Solidarität abhanden kommt, die ihn prägt. Damit kommt es auch unter diesem Gesichtspunkt darauf an, die reale Situation der Ehevertragspartner zu erfassen, sie abzugleichen mit dem Inhalt des Vertrages und an beidem zu bemessen, ob die Regelungen noch als Grundlage für eine partnerschaftliche Beziehung wechselseitiger Verantwortung gelten können.

Nun wird darauf hingewiesen, dass der Solidaritätsgesichtspunkt gerade bei Eheverträgen, die vor Eingehen der Ehe geschlossen würden, nicht greifen könne, da hier noch Verhandlungsgegenstand sei, ob eine solche Solidargemeinschaft überhaupt begründet werden solle, und ein Verzicht auf nacheheliche Solidarität nur etwas betreffe, worauf ohne eine Ehe kein Anspruch bestehe. Richtig daran ist, dass niemand zur Ehe gezwungen werden darf und dass insofern gesetzliche Folgen, die an den Eheschluss geknüpft sind, nicht eintreten, wenn es nicht zur Ehe kommt. Die Eheschließungsfreiheit erhebt sich über jeglichen moralischen Druck, und sofern es ihn geben sollte, garantiert Art. 6 Abs. 1 GG, dass ihm nicht Folge geleistet werden muss. Allein hieraus den Schluss zu ziehen, dass die Nichtjustiziabilität der Entscheidung für und gegen eine Ehe auch den Inhalt des Ehevertrages der richterlichen Kontrolle entzieht, klammert aber aus, dass der Ehevertrag den Entschluss zur Ehe voraussetzt und seine Wirkung erst dann entfalten kann, wenn dieser Entschluss auch umgesetzt worden ist. Gegenstand der vertraglichen Regelung ist also die Ehe, nicht die ihr vorgelagerte

34 Teubner, Ein Fall von struktureller Korruption? in: KritV 2000, S. 388 (396 ff.).

35 Teubner, a.a.O., S. 401. 
Freiheit, sie nicht zu begründen. Insofern kann die Ehevertragsfreiheit nicht mehr herangezogen werden, um eine Freiheit zu unbegrenzter Ehevertragsgestaltung zu rechtfertigen. ${ }^{36}$ Hat ihre Ausübung zur Entscheidung für die Ehe geführt, ist dies für keinen der Ehepartner ein Freifahrschein, seine Interessen einseitig zu Lasten des anderen durchzusetzen.

Nach diesen Ausführungen zu den verfassungsrechtlichen Grenzen und Notwendigkeiten einer Inhaltskontrolle von Eheverträgen werden sich manche von ihnen bestätigt fühlen in ihrer Skepsis, wie mit den vom Verfassungsgericht aufgestellten Vorgaben in der zivilrechtlichen Praxis handhabbar umgegangen werden kann. Dies betrifft zum einen die Frage, ob ein Ehevertrag, der den verfassungsrechtlichen Anforderungen nicht entspricht, über $\S 138$ BGB seine Wirksamkeit verliert oder ob man sich auf ihn oder Teile seines Inhalts wegen $\S 242$ BGB nicht berufen kann. Hier wurde beklagt, dass das Bundesverfassungsgericht nicht deutlicher geworden ist, sondern lediglich darauf verwiesen hat, dass die Grundrechte ihre Wirkungskraft vor allem auch durch die zivilrechtlichen Generalklauseln entfalten, die in ihrem Lichte auszulegen sind. Solche Kritik verwundert, hat doch das Gericht hier gerade die Zurückhaltung geübt, die von ihm ansonsten eingefordert wird: den verfassungsrechtlichen Rahmen zu stecken, die dogmatische Ausfüllung aber der zivilrechtlichen Lehre und den Gerichten zu überlassen. Gerne aber will ich hierzu meine persönliche Auffassung skizzieren. Meines Erachtens kann diese Frage nicht mit einem EntwederOder beantwortet werden. War schon bei Vertragsschluss erkennbar, dass der Ehevertrag seinem Inhalt nach unter Berücksichtigung der Situation der Vertragspartner und der familiären Konstellation, die sie anstreben oder in der sie leben, einen Vertragspartner einseitig belastet, dessen Interessen nicht berücksichtigt und kein solidarisches Miteinander bei Anerkennung der Gleichwertigkeit der in der Ehe zu erbringenden oder erbrachten Leistungen erkennen lässt, dann ist § 138 BGB der Maßstab, an dem die Wirksamkeit des Vertrages zu messen ist. Recht zu geben ist aber auch all denjenigen, die darauf hinweisen, dass sich gerade bei einem auf Dauer angelegten Zusammenleben wie der Ehe Ausgangssituationen, die bei Vertragsschluss vorgelegen haben, verändern können: erhoffte berufliche Karrieren können eintreten oder scheitern, Kinderwünsche können sich realisieren oder zerschlagen, die Aufteilung der Erwerbsund Familienarbeit kann wechseln. ${ }^{37}$ Und weil Eheverträge in der Regel für den Fall des Scheiterns der Ehe abgeschlossen werden, kann der Vertragsinhalt im Lichte der Entwicklung, die die Ehe genommen hat, und der Situation, wie sie sich am Ende der Ehe darstellt, auf ein anderes Kräfteverhältnis der Ehegatten treffen, auf das es nun einwirkt. Führte seine Durchsetzung aufgrund dessen zu einem disparaten Ergebnis, ist es $\S 242$ BGB, der als Prüfmaßstab für eine eventuell notwendige Korrektur einzusetzen ist. Ich bin sicher, dass die zivilistische Rechtsprechung bald eigene Kriterien für die Beurteilung herausgearbeitet hat, ob und wann sich ein Ehevertrag als unwirksam erweist oder die Durchsetzung seiner Wirksamkeit an § 242 BGB scheitert.

36 Vgl. BVerfGE 103, 89 (101 f.).

37 Statt vieler Dauner-Lieb, Reichweite und Grenzen der Privatautonomie im Ehevertragsrecht, in: AcP 201 (2001), S. 295 (325 ff.). 
Bis dahin sollte in der notariellen Praxis das Augenmerk insbesondere darauf gelegt werden, den gewünschten Inhalt eines Ehevertrages abzugleichen mit der Situation, in der sich die Vertragsschließenden befinden und dabei insbesondere mit ihrer familiären Konstellation. Insofern ist es ein erfolgversprechender Weg, Ehetyp-spezifische Vertragstexte weiterzuentwicklen, wie dies schon einige in verdienstvoller Weise unternommen haben, um mit deren Hilfe der Gefahr einer Unwirksamkeit des Ehevertrages zu begegnen. So vielfältig sich eheliches Leben gestalten kann, so vielfältig sind eben auch die Spielräume, die der Ehevertragsfreiheit eröffnet sind, wenn in allen Varianten Freiheit nicht auf Kosten des anderen Ehegatten gehen darf.

Dies auszuloten ist oftmals nicht einfach. Doch hieraus den trotzig anmutenden Schluss zu ziehen, zum Händeweg von der Ehe zu raten, oder gar den Niedergang der Ehe zu beschwören und dies als Konsequenz der Rechtsprechung des Bundesverfassungsgerichts anzuprangern, womit den Frauen ein schlechter Dienst erwiesen worden sei, würden sie nun gerade in schwierigen Situationen weniger häufig geheiratet, scheint mir eine nicht nur übertriebene und realitätsferne Reaktion zu sein, sondern auch eine, die noch von einem Bild gespeist wird, das unter dem Grundgesetz keinen Platz mehr hat: das Bild von der Wohltat des Mannes, die Frau zu heiraten, wofür sie dankbar sein muss.

Zum einen ist der Anteil der Ehen, für die Eheverträge geschlossen werden, mit 10 Prozent noch recht gering und selbst hier finden sich zum Beispiel nur in der Hälfte der Verträge Absprachen zum Versorgungsausgleich und in weniger als einem Viertel der Verzicht auf nachehelichen Unterhalt. Berücksichtigt man zudem, dass die Zahl der auch in der Ehe erwerbstätigen Frauen selbst bei sich einstellenden Kindern in den letzten Jahrzehnten gestiegen ist, dann reduziert sich das Problem der Vertragsdisparität auf einen zwar relevanten, weil schützenswerten, aber doch sehr geringen Teil von Ehen. Wenn das Risiko der Haltbarkeit disparater Eheverträge der einzige Grund wäre, die Ehe nicht einzugehen, müsste man sich um ihren Bestand als Institution deshalb keine Sorge machen.

Zum anderen meine ich, dass es ein guter und verantwortungsvoller Dienst ist, Frauen wie Männer darauf hinzuweisen, eine Ehe nicht unter das Vorzeichen zu stellen, für den Fall ihres Scheiterns den Verlierer schon ausmachen zu wollen. Dass die Ehe keine »ex und hopp«-Veranstaltung, sondern eine enge personale Verantwortungsgemeinschaft ist, zeichnet sie aus und unterscheidet sie von vielen sonstigen Partnerschaften. Wer dies nicht akzeptiert, sollte besser von seiner Eheschließungsfreiheit Gebrauch machen und nicht heiraten. So könnten zwar einzelne Ehen nicht zu Stande kommen. Wen aber trifft dies? Nicht allein die Frau, sondern auch den Mann, denn beiden entgeht die Sicherheit, die aus einer ehelichen Solidarität erwächst. Insofern bin ich nicht nur als ohne Ehevertrag über 30 Jahre glücklich Verheiratete, sondern auch wegen der auch bei jungen Menschen nicht nachlassenden Sehnsucht nach familiärer Geborgenheit und Unterstützung frohen Mutes, dass die Ehe ihre Attraktivität nicht deshalb verliert, weil mit ihr Verantwortung auch für den anderen übernommen wird. So bleibt als Ergebnis festzuhalten: der Ehevertrag kann dabei helfen, die Verantwortlichkeiten der Ehegatten auf deren Bedürfnisse hin maßzuschneidern, er darf die Ehe aber als gleichberechtigte Partnerschaft dieser Verantwortlichkeiten nicht entkleiden. 